\title{
Medicinal Plants Used by Traditional Healers for the Treatment of Various Diseases in Ondae Sub-ethnic of Poso District in Indonesia
}

\author{
Nuning Rahmawati ${ }^{1 *}$, Yuli Widiyastuti ${ }^{1}$, Rahman Purwanto ${ }^{2}$, Siti Sri Lestari ${ }^{3}$, Ikhsan \\ Hi. Amir Sene ${ }^{4}$, Yulianti Bakari ${ }^{5}$
}

\author{
${ }^{1}$ Medicinal Plant and Traditional Medicine Research and Development Center, Central Java, Indonesia \\ ${ }^{2}$ Madani Junior High School, Palu, Central Sulawesi, Indonesia \\ ${ }^{3}$ Graduate students of Biology Faculty, Gadjah Mada University, Yogyakarta, Indonesia \\ ${ }^{4}$ PKMK FKKMK, Gadjah Mada University, Yogyakarta, Indonesia \\ ${ }^{5}$ Biology Faculty, Tadulako University, Palu, Central Sulawesi, Indonesia \\ *Corresponding author. E-mail: nunrahmawati@gmail.com
}

\begin{abstract}
Exploration and inventory of medicinal plants and their use in the community based on local wisdom need to be conducted. This ethno-pharmacological study was undertaken to documenting the medicinal plants and herbal formula utilized by traditional healers in prevention as well as treatment of various diseases in Ondae sub-ethnic of Central Sulawesi of Indonesia. The research was conducted in five selected villages located in Poso District of Central Sulawesi. Traditional healers were selected using purposive sampling based on many inclusion criteria. Data collection was done by direct interviews with traditional healers using a structured questionnaire, observation, and medicinal plants specimen collection. Totally, there are 66 medicinal plant species distributed among 34 plants family used by traditional healers in Onda'e sub-ethnic whereas Euphorbiaceae is the most widespread family used. As of 146 herbal formula are determined among 5 selected traditional healers to prevent and cure ailments. Leaf (34.6\%) is recognized as the most common plant part used followed by herbaceous plant parts $(21.5 \%)$, while flower $(0.6 \%)$ is the least plant parts utilized in Ondae sub-ethnic. A tumor (cancer) and child healthcare are the most general disease handled by traditional healers in Onda'e sub-ethnic. Traditional knowledge of medicinal plants utilization both for preventing and healing diseases still exist in fifth selected villages among Onda'e sub-ethnic of Central Sulawesi especially by traditional healers. Good documentation on the database is expected to be one of the ways to prevent the loss of this local knowledge. Recommendation: Further investigation of laboratory experimental research needs to be conducted to provide scientific data on the safety and efficacy of medicinal plants utilized by traditional healers.
\end{abstract}

Keywords: Ondae sub-ethnic, ethno-pharmacological, local knowledge

\section{INTRODUCTION}

From a total of 40,000 species of medicinal plants in the world, it is known that as many as 30,000 species grow and are in the territory of Indonesia, but only around 7,500 plant species have been identified for their efficacy and pharmacological activities and only 1,200 species of medicinal plants have been utilized as raw material for manufacturing herbal medicine (1).

Information regarding the use of medicinal plants for traditional medicine is known and recognized by most of the world's population. Indigenous traditional knowledge is generally obtained from previous generations (1) and has largely remained undocumented. Cultural modernization has the potential to be one of the causes of the loss of traditional local knowledge that is owned by traditional communities in Indonesia (2).

Ondae is a sub-ethnic of Pamona ethnic group of Poso district located in Central Sulawesi Province. In Central Sulawesi, generally the people of an ethnic group are called as "To", and also for Ondae where the Ondae ethnic community is called To Ondae. The name began with "To" meaning "people of" usually followed by the name of the origin village or some geographic marker (3). With the geographical position of Central Sulawesi which is in the "heart" of Wallacea and identified as one of the centers of biodiversity in the world, this 
region is a unique region rich in endemic flora and fauna, one of which is medicinal plants (4).

Ethno-botany and ethno-pharmacological research data related to medicinal plants and their use in Central Sulawesi are readily available $(2,5,6,7,8,9,10)$ but those that specifically identify medicinal plant utilization in the Ondae sub-ethnic are not yet available. Therefore, exploration and inventory of medicinal plants and their use in the community especially in Ondae subethnic based on local wisdom need to be conducted. This ethno-pharmacological study was undertaken to documenting the medicinal plant and herbal formula utilized by traditional healers in prevention as well as treatment of various diseases in Ondae sub-ethnic of Central Sulawesi of Indonesia.

\section{METHOD}

This research was carrying out in Ondae sub-ethnic, East Pamona sub-District of Poso Regency of Central Sulawesi Province, Indonesia during May 2017. Based on the latitude and longitude, Poso Regency is located at $1^{\circ} 0644^{\prime} 892 "-$ $2^{\circ} 12^{\prime} 53,172^{\prime \prime} \mathrm{SL}$ and $120^{\circ} 05^{\prime} 96^{\prime \prime}-120^{\circ} 52^{\prime} 4,8^{\prime \prime}$ EL with an area of $7112,25 \mathrm{~km}$ or about $11.5 \%$ of Central Sulawesi Province wide (11).

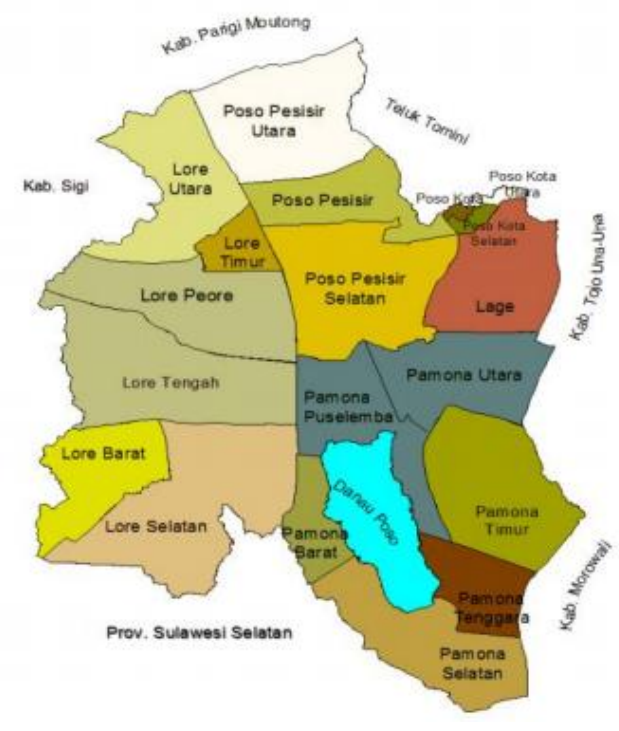

Figure 1. Map of Poso Regency, Central Sulawesi Province, Indonesia where Ondae sub-ethnic of East Pamona subDistrict located

Data collecting was carrying out by interview, observation and medicinal plants sample identification and collection. The interview was conducted using a systematic structured questionnaire to selected traditional health practitioners who met the specified inclusion criteria. This is intended to obtain demographic data of healers, explore information on the ailments overcoming, medicinal plants used, the vernacular name, plant part used, herbal composition, dosage form and the way of preparation.

The traditional healer selection based on purposive sampling method. Information related to the existence of traditional healers was obtained from tribe leaders, religious leaders, communities and community leaders in Ondae subethnic. From the results of coordination with them, it was known that there are as many as seven traditional healers who have knowledge and treatment skills using medicinal plants in the Ondae sub-ethnic, after a further confirmation it was discovered only five traditional healers met the specified inclusion criteria. The specified inclusions criteria including traditional healer who was the native ethnic or enculturated, had both knowledge and expertise on utilizing medicinal plants for healing diseases, the most well-known healers, had the highest number of patients and recognized by the communities.

Data analysis of the relative importance of each plant species was reported as the use value (UV) and it was calculated using the following formula (1):

$$
\mathrm{UV}=\sum \mathrm{U} / \mathrm{n}
$$

Where UV is the use value of a species, $\mathrm{U}$ is the number of use reports cited by each informant for a given plant species and $\mathrm{n}$ is the total number of informants interviewed for a given plant. The UV is helpful in determining the plants with the most frequently indicated in an ailment treatment. UV will be higher for the species with more cited indication reports and become lower for species with less use reports.

\section{RESULTS AND DISCUSSION}

The survey revealed all selected traditional healers came from rural areas in East Pamona sub-district of Poso District, Central Sulawesi Province. The traditional healer characteristics included gender, age, level of education and patient number per month were completely shown in table 1 .

Table 1. Characteristic of selected traditional healers as informants

\begin{tabular}{|c|c|c|c|c|c|}
\hline \multirow{2}{*}{ Characteristic } & \multicolumn{5}{|c|}{ Informants } \\
\hline & I & II & III & IV & v \\
\hline $\begin{array}{l}\text { Gender } \\
\text { Age (years old) }\end{array}$ & $\begin{array}{c}\text { Male } \\
84\end{array}$ & $\begin{array}{c}\text { Female } \\
48\end{array}$ & $\begin{array}{c}\text { Female } \\
60\end{array}$ & $\begin{array}{c}\text { Female } \\
47\end{array}$ & $\begin{array}{c}\text { Female } \\
43\end{array}$ \\
\hline Education & $\begin{array}{l}\text { Elementary } \\
\text { school }\end{array}$ & $\begin{array}{l}\text { Elementary } \\
\text { school }\end{array}$ & $\begin{array}{l}\text { Junior high } \\
\text { school }\end{array}$ & $\begin{array}{l}\text { Junior high } \\
\text { school }\end{array}$ & $\begin{array}{c}\text { Elementary } \\
\text { school }\end{array}$ \\
\hline Hometown & Taripa village & Didiri village & $\begin{array}{c}\text { Poleganyara } \\
\text { village }\end{array}$ & Kelei village & $\begin{array}{c}\text { Matialemba } \\
\text { village }\end{array}$ \\
\hline $\begin{array}{l}\text { Patient number } \\
\text { per month }\end{array}$ & 28 & 8 & 25 & 24 & 3 \\
\hline
\end{tabular}

The ailments treated, herbs formula and composition, medicinal plant used, medicinal plant sources, vernacular name, plants growth form, plant parts used, preparation and the route of administration data collected among selected traditional healers, identified botanically scientific name and plant have been summarized in Table 2. It was noted that in most cases, traditional healers in Ondae sub-ethnic tend to prepare their herbal formula in single compound rather than in combining formula. Herbs formula prepared 
from more than one plant (mixtures) made up $23.02 \%$ while those from single plants made up $76.98 \%$.

Table 2. Medicinal plants used by traditional healers in Ondae sub-ethnic

\begin{tabular}{|c|c|c|c|c|c|c|}
\hline Family & $\begin{array}{l}\text { Vernacular } \\
\text { name }\end{array}$ & Scientific name & $\begin{array}{c}\text { Use } \\
\text { valu } \\
\text { e }\end{array}$ & $\begin{array}{l}\text { Growth } \\
\text { form }\end{array}$ & Part used & Ailments treated \\
\hline \multirow[t]{3}{*}{ Acantaceae } & Pica Beling & Strobilanthes crispa & 0.2 & Shrub & $\begin{array}{l}\text { Herbaceous } \\
\text { part }\end{array}$ & Kidney disease \\
\hline & Sive & $\begin{array}{l}\text { Graptophyllum } \\
\text { pictum (L.) Griff }\end{array}$ & 0.6 & Herb & Leaf & $\begin{array}{l}\text { Kidney disease, } \\
\text { Appendicitis, cyst }\end{array}$ \\
\hline & Kince Mbeling & Strobilanthes crispa & 0.2 & Shrub & Leaf & Kidney disease \\
\hline Anacardiaceae & Taripa & Mangifera indica $\mathrm{L}$. & 0.2 & Tree & Leaf & Hypertension \\
\hline Annonaceae & Sirikaya & Annona muricata $\mathrm{L}$. & 0.6 & Tree & Leaf & $\begin{array}{l}\text { Tumor/cancer, } \\
\text { magical and spiritual } \\
\text { healing, } \\
\text { hypercholesterolemia }\end{array}$ \\
\hline Apiaceae & Soderei & Apium graveolens $\mathrm{L}$. & 0.2 & Herb & Leaf & Hypertension \\
\hline \multirow[t]{2}{*}{ Arecaceae } & Enau & Arenga pinnata & 0.2 & Tree & Root & Diabetes \\
\hline & Mamongo & Areca catechu L. & 0.6 & Tree & Root, pulp & $\begin{array}{l}\text { Diabetes, fever, } \\
\text { kidney disease }\end{array}$ \\
\hline \multirow[t]{3}{*}{ Asteraceae } & Mbula-mbila & $\begin{array}{l}\text { Ageratum conyzoides } \\
\text { L. }\end{array}$ & 0.6 & Herb & $\begin{array}{l}\text { Root, } \\
\text { herbaceous } \\
\text { part }\end{array}$ & Stomachache, cough \\
\hline & Sambung nyawa & $\begin{array}{l}\text { Ginura procumbens } \\
\text { (Blume.) Miq. }\end{array}$ & 0.2 & & Leaf & Wound healing \\
\hline & Ewomawao & $\begin{array}{l}\text { Ageratum conyzoides } \\
\text { L. }\end{array}$ & 0.4 & Herb & $\begin{array}{l}\text { Herbaceous } \\
\text { part, Other } \\
\text { parts }\end{array}$ & $\begin{array}{l}\text { Fever, pre post- } \\
\text { partum treatment }\end{array}$ \\
\hline Clusiaceae & Manggis & $\begin{array}{l}\text { Garsinia mangostana } \\
\text { L. }\end{array}$ & 0.2 & Tree & Rind & Hypercholesterolemia \\
\hline Convolvulaceae & Spesies A & Ipomea sp. & 0.2 & Liana & Leaf & Appendicitis \\
\hline Costaceae & Tawoo' & Costus spiralis & 0.6 & Herb & $\begin{array}{l}\text { Stem bark, } \\
\text { stem }\end{array}$ & Sore throat, Fever \\
\hline Crassulaseae & Pakumba & $\begin{array}{l}\text { Bryophyllum } \\
\text { pinnatum }\end{array}$ & 0.2 & Herb & Leaf & Kidney disease \\
\hline Cucurbitaceae & Paria & $\begin{array}{l}\text { Momordica charantia } \\
\text { L. }\end{array}$ & 0.2 & Herb & Leaf & Cough \\
\hline Cyperarceae & Tetari & Cyperus rotundus $\mathrm{L}$. & 0.2 & Herb & Other parts & Urolithiasis \\
\hline \multirow[t]{2}{*}{ Euphorbiaceae } & Tai Kando & Euphorbia hirta & 0.4 & Herb & Other parts & Appendicitis \\
\hline & Lepati & $\begin{array}{l}\text { Aleurites moluccanus } \\
\text { (L.) Wild }\end{array}$ & 0.4 & Tree & Seed & Cosmetic, diarrhea \\
\hline
\end{tabular}




\begin{tabular}{|c|c|c|c|c|c|c|}
\hline & Gedi & Abelmoschus manihot & 0.2 & Tree & $\begin{array}{l}\text { Herbaceous } \\
\text { part }\end{array}$ & Kidney disease \\
\hline & Balacae & Jatropha curcas L. & 0.2 & Tree & Leaf & Bone fracture \\
\hline & Tomene & Jatropha curcas L. & 0.2 & Tree & Leaf & Tumor/cancer \\
\hline & Kasubi & Manihot esculenta & 0.2 & Tree & Leaf & gastritis \\
\hline Fabaceae & Tambaole & Cassia alata $\mathrm{L}$. & 0.6 & Tree & Leaf & $\begin{array}{l}\text { Gastritis, skin disease, } \\
\text { dyspepsia }\end{array}$ \\
\hline Iridaceae & Pia manu & $\begin{array}{l}\text { Eleutherine bulbosa } \\
\text { (Mill.) Urb }\end{array}$ & 0.2 & Herb & Bulb & Tumor/cancer \\
\hline \multirow[t]{5}{*}{ Lamiaceae } & Ta'inyara & Hyptis capitata Jacq. & 1.0 & Herb & $\begin{array}{l}\text { leaf, } \\
\text { herbaceous } \\
\text { part }\end{array}$ & $\begin{array}{l}\text { Stomachache, } \\
\text { tumor/cancer, cyst }\end{array}$ \\
\hline & $\begin{array}{l}\text { Jumbi Nggaru', } \\
\text { kumis kucing }\end{array}$ & $\begin{array}{l}\text { Orthosiphon aristatus } \\
\text { (Blume) Miq. }\end{array}$ & 1.4 & Herb & $\begin{array}{l}\text { Herbaceous } \\
\text { part, Other } \\
\text { parts }\end{array}$ & $\begin{array}{l}\text { Urolithiasis, } \\
\text { Appendicitis, kidney } \\
\text { disease, liver disease }\end{array}$ \\
\hline & Mayana & Coleus hybridus & 0.2 & Herb & $\begin{array}{l}\text { Herbaceous } \\
\text { part }\end{array}$ & Tumor/cancer \\
\hline & Ta'inyara Kodi & Hyptis capitata Jacq. & 0.2 & Herb & Root & Stomachache \\
\hline & Ta'inyara Bose & $\begin{array}{l}\text { Hyptis rhomboidea } \\
\text { Jacq. }\end{array}$ & 0.2 & Herb & Root & Stomachache \\
\hline Lauraceae & Pakanangi & Cinnamomum verum & 0.2 & Tree & Stem bark & Bone fracture \\
\hline Liliaceae & Bawang Merah & $\begin{array}{l}\text { Allium ascalonicum } \\
\text { L. }\end{array}$ & 0.4 & Herb & Bulb & $\begin{array}{l}\text { Bone fracture, } \\
\text { anthelminthiasis }\end{array}$ \\
\hline \multirow[t]{2}{*}{ Loranthaceae } & $\begin{array}{l}\text { Pomuyan } \\
\text { Entonci }\end{array}$ & $\begin{array}{l}\text { Macrosolen } \\
\text { cochinchinensis L. }\end{array}$ & 0.4 & $\begin{array}{c}\text { Plant } \\
\text { parasites }\end{array}$ & $\begin{array}{l}\text { Leaf, } \\
\text { herbaceous } \\
\text { part }\end{array}$ & $\begin{array}{l}\text { Liver disease, } \\
\text { tumor/cancer }\end{array}$ \\
\hline & Pomuya Tonci & Loranthus sp. & 0.2 & $\begin{array}{c}\text { Plant } \\
\text { parasites }\end{array}$ & $\begin{array}{l}\text { Herbaceous } \\
\text { part }\end{array}$ & $\begin{array}{l}\text { Tumor/cancer, liver } \\
\text { disease }\end{array}$ \\
\hline Mackinlayaceae & Tele-tele & Centella asiatica $\mathrm{L}$. & 0.6 & Herb & Other parts & Appendicitis, cyst, \\
\hline \multirow[t]{5}{*}{ Malvaceae } & Rabuate & Sida rhombifolia & 0.2 & Herb & Pulp & $\begin{array}{l}\text { Pre-post-partum } \\
\text { treatment }\end{array}$ \\
\hline & Rosela & $\begin{array}{l}\text { Hibiscus sabdariffa } \\
\text { L. }\end{array}$ & 0.2 & Herb & Leaf & Hyperuricemia \\
\hline & Coklat & Theobroma cacao L. & 0.4 & Tree & Fruit, leaf & $\begin{array}{l}\text { Boils, anti- } \\
\text { inflammation }\end{array}$ \\
\hline & Kapas & $\begin{array}{l}\text { Gossypium hirsutum } \\
\text { L. }\end{array}$ & 0.4 & Tree & Leaf & $\begin{array}{l}\text { Heart disease, } \\
\text { tumor/cancer }\end{array}$ \\
\hline & Soklat & Theobroma cacao & 0.2 & Tree & Fruit & Boils \\
\hline Muntingiaceae & Gersen & $\begin{array}{l}\text { Muntingia calabura } \\
\text { L. }\end{array}$ & 0.4 & Tree & $\begin{array}{l}\text { leaf, } \\
\text { herbaceous } \\
\text { part }\end{array}$ & $\begin{array}{l}\text { Hypercholesterolemia, } \\
\text { diabetes }\end{array}$ \\
\hline Musaceae & Loka & Musa paradisiaca L. & 0.4 & Tree & Leaf & $\begin{array}{l}\text { Diabetes, wound } \\
\text { healing }\end{array}$ \\
\hline
\end{tabular}




\begin{tabular}{|c|c|c|c|c|c|c|}
\hline & Loka Tunu & $\begin{array}{l}\text { Musa x paradisiaca } \\
\text { L. }\end{array}$ & 0.4 & Tree & Other parts & $\begin{array}{l}\text { Bone fracture, liver } \\
\text { disease }\end{array}$ \\
\hline \multirow[t]{2}{*}{ Myrtaceae } & Cingke & $\begin{array}{l}\text { Syzygium aromaticum } \\
\text { (L.) Merr. \& Perry }\end{array}$ & 0.2 & Tree & Seed & Other diseases \\
\hline & Jambu & Psidium guajava $\mathrm{L}$. & 0.4 & Tree & Leaf & Stomachache \\
\hline \multirow[t]{2}{*}{ Phyllanthacea } & Simpojuyu & Phyllanthus ninuri $\mathrm{L}$. & 0.2 & Herb & $\begin{array}{l}\text { Herbaceous } \\
\text { part }\end{array}$ & Cough \\
\hline & Dukun Anak & Phyllanthus ninuri L. & 0.6 & Herb & Other parts & $\begin{array}{l}\text { Appendicitis, kidney } \\
\text { disease, cyst }\end{array}$ \\
\hline \multirow[t]{2}{*}{ Piperaceae } & Nepo & Piper umbellatum L. & 0.4 & Perdu & Leaf, root & Tumor/cancer \\
\hline & Bou-Bou & $\begin{array}{l}\text { Peperomia pellucida } \\
\text { L. Kunth }\end{array}$ & 0.4 & Herb & Other parts & $\begin{array}{l}\text { Hypercholesterolemia, } \\
\text { hyperuricemia }\end{array}$ \\
\hline \multirow[t]{3}{*}{ Poaceae } & Timbowane & $\begin{array}{l}\text { Cymbopogon citratus } \\
\text { (DC,) }\end{array}$ & 0.2 & Herb & Stem & Bone fracture \\
\hline & Bulu Batu & Bambusa spp. & 0.2 & Tree & leaf & Cold \\
\hline & Lee & $\begin{array}{l}\text { Imperata cylindrica } \\
\text { L. Raeush }\end{array}$ & 0.6 & Herb & $\begin{array}{l}\text { Root, } \\
\text { herbaceous } \\
\text { part }\end{array}$ & $\begin{array}{l}\text { Liver disease, } \\
\text { tumor/cancer, } \\
\text { Appendicitis }\end{array}$ \\
\hline Portulacaceae & Luangkando & Portulaca tuberosa & 0.2 & Herb & Other parts & Appendicitis \\
\hline \multirow[t]{2}{*}{ Rosaceaea } & Lokaya mbeyo & Rubus idaeus $\mathrm{L}$. & 0.4 & Shrub & Leaf, root & Tumor/cancer, cyst \\
\hline & Lokaya Baula & Rubus idaeus $\mathrm{L}$. & 0.2 & Shrub & Root & Tumor/cancer \\
\hline Rutaceae & Lemo Polea & Cytrus hystrix & 0.2 & Tree & $\begin{array}{l}\text { Herbaceous } \\
\text { part }\end{array}$ & Tumor/cancer \\
\hline \multirow[t]{3}{*}{ Solanacea } & Tomate & $\begin{array}{l}\text { Solanum } \\
\text { lycopersicum }\end{array}$ & 0.6 & Herb & $\begin{array}{l}\text { Herbaceous } \\
\text { part }\end{array}$ & $\begin{array}{l}\text { Pre post-partum } \\
\text { treatment, Fever }\end{array}$ \\
\hline & Palola Yopo & Solanum torvum & 0.2 & Perdu & Root & Diabetes \\
\hline & Lada & Capsicum anпиит $\mathrm{L}$. & 0.4 & Herb & Stem & Fever, asthma \\
\hline \multirow[t]{2}{*}{ Verbenaceae } & Kajungkuluri & Lantana camara & 0.2 & Shrub & $\begin{array}{l}\text { Herbaceous } \\
\text { part }\end{array}$ & gastritis \\
\hline & Iku Valesu & $\begin{array}{l}\text { Stachytarpheta } \\
\text { jamaicensis (L) Vhal }\end{array}$ & 0.6 & Herb & Other parts & $\begin{array}{l}\text { Appendicitis, kidney } \\
\text { disease, cyst }\end{array}$ \\
\hline \multirow[t]{3}{*}{ Zingiberaceae } & Temulawak & $\begin{array}{l}\text { Curcuma } \\
\text { zanthorrhiza Roxb }\end{array}$ & 0.2 & Herb & Rhizome & Liver disease \\
\hline & Kuni & Curcuma mangga $\mathrm{L}$. & 0.2 & Herb & Rhizome & Wound healing \\
\hline & Kuni & Curcuma longa $\mathrm{L}$. & 0.8 & Herb & Rhizome & $\begin{array}{l}\text { Child health care, } \\
\text { hemorrhoid, wound } \\
\text { healing, cough, } \\
\text { gastritis }\end{array}$ \\
\hline
\end{tabular}

In this study, as of 66 plant species were documented from selected traditional healers in Ondae sub-ethnic of Poso Regency which distributed within 34 families whereas Euphorbiaceae and Lamiaceae had the highest number of 
From this present study, Orthosiphon aristatus L was determined as the leading species utilized by traditional healers. Most medicinal plant species were harvested for their leaves $(33.70 \%)$ followed by herbaceous part $(23.20 \%)$ and root $(9.39 \%)$ to prepare remedies (Figure 3 ). Flower, rind and pulp were the least common plant part used.

Table 3. Number of formula used by informants for each ailments

\begin{tabular}{|c|c|c|c|c|c|c|}
\hline \multirow{2}{*}{ Ailments } & \multicolumn{5}{|c|}{ Number of formula used by Informants } & \multirow{2}{*}{$\begin{array}{c}\text { Total } \\
\text { formula }\end{array}$} \\
\hline & I & II & III & IV & $\mathbf{v}$ & \\
\hline Child health treatment & 3 & 9 & 4 & - & 3 & 19 \\
\hline Undefined diseases & 3 & 3 & 3 & 6 & - & 15 \\
\hline Tumor/cancer & 6 & 4 & - & - & 2 & 12 \\
\hline Kidney disorders & 1 & 5 & 1 & 2 & 1 & 10 \\
\hline Cough & - & 2 & 1 & 2 & 2 & 7 \\
\hline Hypercholesterolemia & 2 & 4 & 1 & - & - & 7 \\
\hline $\begin{array}{l}\text { Pre and postpartum } \\
\text { treatment }\end{array}$ & 1 & 2 & 1 & 1 & 2 & 7 \\
\hline Gastritis & - & 4 & - & - & 1 & 5 \\
\hline Hypertension & - & 2 & 1 & - & 1 & 4 \\
\hline Diabetes & 1 & 3 & - & - & - & 4 \\
\hline Wound healing & 2 & 1 & - & - & 1 & 4 \\
\hline Diarrhea & 1 & 1 & 1 & 1 & - & 4 \\
\hline Stomachache & 1 & 1 & 1 & - & 1 & 4 \\
\hline Fever & - & 3 & - & - & - & 3 \\
\hline Venereal treatment & 1 & - & 1 & . & 1 & 3 \\
\hline Rheumatics & 1 & 1 & - & - & 1 & 3 \\
\hline Dental care & - & 1 & - & - & 2 & 3 \\
\hline Liver disease & 1 & 1 & 1 & - & - & 3 \\
\hline Appendicitis & - & 1 & 1 & 1 & - & 3 \\
\hline Bone fracture & - & 1 & - & - & 1 & 2 \\
\hline Flu & - & 2 & - & - & - & 2 \\
\hline Helminthiasis & 1 & 1 & - & - & - & 2 \\
\hline Asthma & - & 2 & - & - & - & 2 \\
\hline Hemorrhoids & - & 2 & - & - & - & 2 \\
\hline Boils & 1 & 1 & - & - & - & 2 \\
\hline Antiinflammation & - & - & - & - & 1 & 1 \\
\hline Diuretics & - & 1 & - & - & - & 1 \\
\hline Hernia & - & - & 1 & - & - & 1 \\
\hline Anemia & - & 1 & - & - & - & 1 \\
\hline Magical spiritual & 1 & - & - & - & - & 1 \\
\hline $\begin{array}{l}\text { healing } \\
\text { Malaria }\end{array}$ & - & 1 & - & - & - & 1 \\
\hline Nose bleeding & - & 1 & - & - & . & 1 \\
\hline Neonatal treatment & - & 1 & - & - & - & 1 \\
\hline Cosmetics & - & 1 & - & - & - & 1 \\
\hline Skin disease & - & 1 & - & - & - & 1 \\
\hline Ear disease & - & - & 1 & - & - & 1 \\
\hline Constipation & - & 1 & - & - & - & 1 \\
\hline Tuberculosis & - & - & - & 1 & - & 1 \\
\hline Thypus & - & - & - & - & 1 & 1 \\
\hline Fomula per informants & 27 & 65 & 19 & 14 & 21 & 146 \\
\hline
\end{tabular}

Of 146 herbs formula documented from five selected traditional healers in Ondae sub-ethnic of Poso District, it revealed whether 2 nd informant had the most herbs formula followed by 1 st informant as shown in Table 3. Otherwise, the 1 st informant reported to have more patient than patient number of 2 nd informant monthly. The administration route of herbs formula consisted of three category, they are internal, external and combination. The most common route of provision was internal (69\%) followed by external (28\%) and combination route $(3 \%)$. From this current ethnopharmacological study, child health treatment was known as the most frequent ailment handled by traditional healers followed by tumor/cancer and kidney disorders as shown in Figure 5 .

Figure 4. Plant parts used by informants 


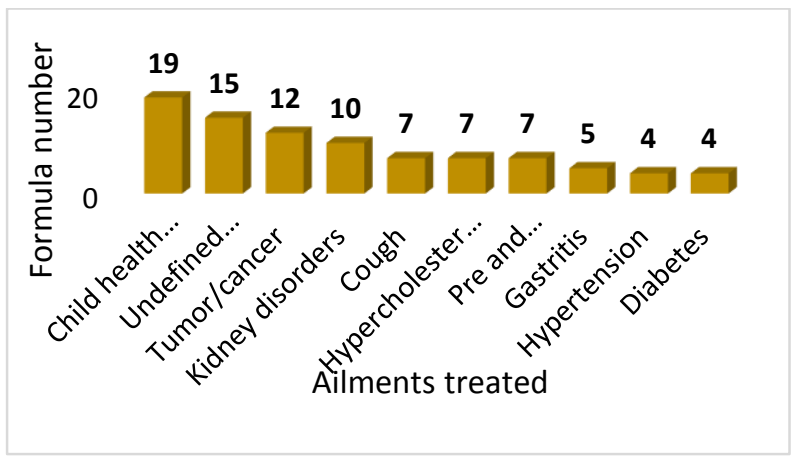

Figure 5 . The ten leading ailments treated by informants

\section{Discussion}

This ethno-pharmacological study was undertaken to documenting the medicinal plants and herbal formula utilized by traditional healers in prevention as well as treatment of various diseases in Ondae sub-ethnic of Central Sulawesi. Plants are very pivotal resources in both healthcare and pharmaceutical drugs production (13). All informants age in Ondae sub-ethnic were more than 40 years old and had low level of formal education. This is in line with result stated by Xavier et al. (2014) (14) whether most of informant in Kani tribes of South India did not have formal education and most of them lives in the region. Most of older generation with low formal education tend to have more knowledge within medicinal plants usage (15). Research conducted by Nyundu and Naido (16) shows that South African youth do not place traditional medicine as a very important part of their lives, they will choose traditional medicine when they do not get satisfaction with conventional treatment. Several factors affect their knowledge and attitudes towards traditional medicine including age, education level and the surrounding environment (17). The other factors on utilizing traditional medicine are the belief of the effectiveness of traditional medicine as an inheritance, saves time and money and is easier to obtain (6) also incomparable advantages, such as abundant clinical experiences, and their unique diversity of chemical structures and biological activities (18). Nevertheless, accessibility, low cost, effectiveness and belief in safeness and tradition influence the use of the medicine (19). Good documentation is needed to maintain historical records knowledge on medicinal plants before they are extinct forever (20). This study revealed the presence and the utilization of 66 species distributing in 34 families and 146 herbal formula in Ondae sub-ethnic.

From this study, Euphorbiaceae represents the highest number of species utilized by traditional healers in Ondae sub-ethnic for therapeutic goals. This is in line with previously studies reported the usage of medicinal plants from the family Euphorbiaceae by the Pekurehua tribe in Wuasa and Kaduwaa Village, Central Sulawesi to overcoming fever, mouth sores, wounds and back pain, they are Euphorbia hirta L, Acalypha indica L and Jatropha curcas L (7). Likewise, research conducted in the Kaili Ija ethnic group in Bora village, Sigi Biromaru sub-district,
Sigi Regency, Central Sulawesi reported that five species of the Euphorbiaceae family plant were used as traditional medicine, namely Jatropha curcas L, Acalypha indica L, Euphorbia hirta L, Manihot utilissima Pohl and Phyllanthus niruri L (6). Nevertheless, these are contrary to the research results submitted by Kadir et al. (15), Odhiambo et al. (21) and Gairola et al. (22) whereas Asteraceae is recognized as the most utilized family. The high utilization rate of the Euphorbiaceae family is related to the number of species from that family which reaches 7500 species distributed in 300 genera where each species has a diverse range of pharmacological activities (23).

O. aristatus from Lamiaceae family showed the highest percentage $(6.14 \%)$ as well as highest use value ( $U V=1.4)$ of species exerted by informants of Ondae sub-ethnic. Most of treaditional healers applied herbaceous part of this plant for healing such ailments like kidney disease, urolithiasis, appendicitis and liver disease. This is in accordance with Silalahi (2019) in which O. aristatus from genera Orthosiphon was used in traditional medicine to prevent many disease such as diabetes mellitus, kidney stones, edema, rheumatism, hepatitis, hypertension and jaundice. It has been reported whether some of secondary metabolites in the O. stamineus which contributing in therapeutic effect are terpenoids, phenols, ispenimoids, flavonoids, benzochromes and organic acid derivatives (24).

Leaves were the most preferred plant part used in the study area with a percentage as of $33.70 \%$, followed by herbaceous parts, root and other parts. Higher preference of leaves utilized could be due to its higher availability in the area as well as its established pharmaceutical potency. Leaves were the most widely used part of the plant for treatment by the Kaili ethnic community of Central Sulawesi (6). Many studies have been conducted in various regions also showed the dominance of leaf organs in the use of traditional medicine $(7,20)$. This is quietly different with study conducted by Shresta et al. (2016) (12) in Nepal, whereas root was the most utilized parts followed by leaves and other plant parts. According to traditional health practitioner, leaves have a high level of effectiveness as drugs (5). Traditional healers in Ondae sub-ethnic obtained and collected plant materials mainly from the house yard $(76.8 \%)$ and the surrounding forest $(12.15 \%)$. Forest was recognized as the center of healing illnesses of indigenous people of Tau Taa Vana in Tojo Una-Una Central Sulawesi (8). Communities surrounding forest areas uses the existing medicinal plants for healing purposes based on knowledge passed down from generations (9). Topography, climate, culture and ethnic are some factor affecting diversity of plant species in the home garden. It was also stated whether plants had more diversity during dry season than rainy season in the home garden (25).

Child health diseases were the most frequent ailments healed by traditional healers in Ondae sub-ethnic of Poso District of Central Sulawesi Province followed by other disease, tumor/cancer and kidney disease. This is in accordance with many previous researches reported. Study of 400 children with the age of 12 to 59 months conducted in Jamnagar District, India exhibited acute respiratory infection and diarrhea as two leading disease in child with 
[4] Pitopang R, Ramawangsa PA. Potencial of ethnobotanical studies in Central Sulawesi Indonesia. Online J Nat Sci. 2016;5(2):111-31.

Pneumonia, diarrhea, measles, tetanus and polio were categorized as five child killer diseases in Nigeria and the analysis exhibited the close connection between under-five child mortality rate with incidence of pneumonia and diarrhea (27). Meanwhile, data on ten diseases with the highest number of cases in Poso district in 2017 included upper respiratory infections, gastritis, arthritis, hypertension, malaria, diarrhea, hypotension, tonsillitis, skin diseases and accidents (11). Report of public health service of Singgani, Palu City in 2016 showed an increase of acute respiratory infections as of 3,270 cases on children at mine area. The number of Central Sulawesi people with acute respiratory infection (ARI) was increased significantly (46.1\%) during 2012 to 2016 (28). Prevalence of ARI was significantly less in exclusively breastfed children compared to not exclusively breastfed children $(\mathrm{p}=0.002)$ (29). Children are generally more susceptible to diseases.

\section{CONCLUSION}

In conclusion, the study clearly demonstrated that traditional healers in Ondae sub-ethnic plays important role on treating many ailments utilizing medicinal plants with the total number of 66 species classified into 34 families, distributing within 146 herbal formula. The species with the highest use value was Orthosiphon aristatus and plant family contributed the most species number was Euphorbiaceae. The efficacy and safety of all reported medicinal plants needs to be further evaluated for phytochemical and pharmacological studies to provide scientific database as an alternative on drugs development.

\section{ACKNOWLEDGMENT}

The authors are fully grateful to the Ondae sub-ethnic of Poso Regency, Central Sulawesi Province especially to all interviewed traditional healers for sharing their valuable indigenous knowledge on traditional medicine using medicinal plants. The authors are also thankful to Medicinal Plant and Traditional Medicine Research and Development Center (MPTMRDC, NIHRD) for the financial support and also thanks to RISTOJA full teams for all substantial and technical supports.

\section{REFERENCES}

[1] Salim Z, Munadi E. Info komoditi tanaman obat. Badan Pengkaj dan Pengemb Perdagang. 2017;1-8.

[2] Islami MY, Ibrahim $\mathrm{N}$, Nugrahani AW. Ethnomedicinal study of Kaili Moma tribe in Kulawi Subdistrict, Sigi Regency, Central Sulawesi. Galen J Pharm. 2017;3(1):27-33.

[3] Schrauwers A. Houses of worship in Central Sulawesi: Precedence, hierarchy \& class in the development of house ideology. J Soc Anthropol Comp Sociol. Taylor \& Francis; 2017;26(4):33354. Understanding and Utilization of Medicinal Plants by Local Community Tompu District of Kaili, Sigi Biromaru, Central Sulawesi. Biosaintifika. 2016;8(1):1-11.

[6] Megawati, Anam S, Pitopang R. Studi etnobotani tumbuhan obat pada masyarakat suku Kaili Ija di Desa Bora Kecamatan Sigi Biromaru Kabupaten Sigi Sulawesi Tengah. Biocelebes. 2016;10(1):7690.

[7] Adhil A, Iqbal M, Pitopang R. Kajian Etnobotani Suku Euphorbiaceae Yang Dimanfaatkan Oleh Suku Pekurehua Di Desa Wuasa Dan Kaduwaa Kecamatan Lore Utara Kabupaten Poso Sulawesi Tengah Study of Ethnobotany Family Euphorbiaceae Used by Pekurehua Tribe in Wuasa and Kaduwaa, North Lore ,. Nat Sci J Sci Technol. 2019;8(1):51-60.

[8] Humaedi MA. Tradisi pelestarian hutan masyarakat adat tau taa vana di tojo una-una sulawesi tengah (. J Penelit Hutan dan Konserv Alam. 2014;11(1):91111.

[9] Kurniawan T, Sri N, Toknok B. Keanekaragaman jenis tumbuhan obat pada kawasan hutan lindung di Desa Tindoli Kecamatan Pamona Tenggara Kabupaten Poso. War Rimba. 2014;2(1):120-8.

[10] Praptiwi P, Raunsai M, Wulansari D, Fathoni A, Agusta A. Antibacterial and antioxidant activities of endophytic fungi extracts of medicinal plants from Central Sulawesi. J Appl Pharm Sci. 2018;8(8):6974.

[11] Anonim. Poso Regency in Figures. 2018;

[12] Shrestha N, Shrestha S, Koju L, Shrestha KK, Wang Z. Medicinal plant diversity and traditional healing practices in eastern Nepal. J Ethnopharmacol [Internet]. Elsevier; 2016; Available from: http://dx.doi.org/10.1016/j.jep.2016.07.067

[13] Souza ENF, Williamson EM, Hawkins JA, Vale IT, Stafford GI. Which Plants Used in Ethnomedicine Are Characterized? Phylogenetic Patterns in Traditional Use Related to Research Effort. 2018;9(June):1-12.

[14] Xavier TF, Kannan M, Lija L, Auxillia A, Rose AKF, Kumar SS. Ethnobotanical study of Kani tribes in Thoduhills of Kerala, South India. J Ethnopharmacol [Internet]. Elsevier; 2014;152(1):78-90. Available from: http://dx.doi.org/10.1016/j.jep.2013.12.016

[15] Kadir MF, Sayeed MS, Setu NI, Mostafa A, Mia M. Ethnopharmacological survey of medicinal plants used by traditional health practitioners in Thanchi , Bandarban Hill Tracts, Bangladesh. J Ethnopharmacol [Internet]. Elsevier; 2014;1-15. Available

from:
[5] Ifandi S, Jumari, Suedy SWA. Knowledge 
Agric Environ. 2017;15(3\&4):115-22.

http://dx.doi.org/10.1016/j.jep.2014.05.043

[16] Nyundu T, Naidoo K. Traditional healers, their services and the ambivalence of South African youth. Coommonwealth Youth Dev. 2018;14(1):144-55.

[17] Napirah MR, Rahman A, Tony A. Faktor-faktor yang berhubungan dengan pemanfaatan pelayanan kesehatan di wilayah kerja Puskesmas Tambarana Kecamatan Posos Pesisir Utara Kabupaten Poso. J Pengemb Kota JPK. 2016;4(1):29-39.

[18] Yuan H, Ma Q, Ye L, Piao G. The Traditional Medicine and Modern Medicine from Natural Products. Molecules. 2016;21:1-18.

[19] Sarki ZM, Danjuma MH. Socio-demographic Factors and Utilization of Traditional Medicine in Kazaure Town, Jigawe State, Nigeria. Int J Emerg Knowl. 2015;3(1):9-20.

[20] Jamshidi-kia F, Lorigooini Z, Amini-khoei H. Medicinal plants: Past history and future perspective. J HerbMed Pharmacol. 2018;7(1):1-7.

[21] Odhiambo JA, Lukhoba CW, Dossaji SF. Evaluation of herbs as potential drugs/medicines. African J Tradit Complement Altern Med. 2011;8(S):144-51.

[22] Gairola S, Sharma J, Bedi YS. A cross-cultural analysis of Jammu, Kashmir and Ladakh (India) medicinal plant use. J Ethnopharmacol [Internet]. Elsevier; 2014; Available from: http://dx.doi.org/10.1016/j.jep.2014.06.029

[23] Rahman AHMM, Akter M. Taxonomy and Medicinal Uses of Euphorbiaceae (Spurge) Family of Rajshahi, Bangladesh. Res Plant Sci. 2013;1(3):74-80.

[24] Silalahi M. Orthosiphon stamineus Benth (Uses and Bioactivities). Indones J Sci Educ. 2019;3(1):26-33.

[25] Silalahi M. The ethnobotanical study of edible and medicinal plants in the home garden of Batak Karo sub-ethnic in North Sumatra, Indonesia. 2018;19(1):229-38.

[26] Bhavsar S, Sarkar A. Assesment od common childhood disease in 1-5 years age group children and determination of knowledge, health care practices \& health seeking behavior of parents in Jamnagar District. Glob J Res Anal. 2017;6(4):525.

[27] Nafiu LA, Hamidu UW. Prevalence of Five-ChildKiller Diseases and Under-Five Mortality in Adamawa Prevalence of Five-Child-Killer Diseases and Under-Five Mortality in Adamawa State, Nigeria. KIU J Soc Sci. 2018;3(1):13-20.

[28] Basir-cyio M, Inoue T, Anshary A, Kawakami T, Rahman N, Rusydi M, et al. Impact of the traditional gold mine management on public health and agricultural land: A Study of traditional gold mining in Poboya, Sausu and Impact of the traditional gold mine management on public health and agricultural land : A Study of traditional. J Food,
[29] Kadirvelu U, Sharma N, Velu M, Kohli C. Epidemiology of childhood diseases in an urban resettlement colony in Delhi. Clin Epidemiol Glob Heal [Internet]. INDIACLEN; 2016;4(4):176-80. Available from: http://dx.doi.org/10.1016/j.cegh.2016.05.001. 